\title{
Fibre imaging bundles for full-field optical coherence tomography
}

\author{
H D Ford and R P Tatam \\ Engineering Photonics Group, School of Engineering, Cranfield University, Cranfield, \\ Bedford MK43 0AL, UK \\ E-mail: r.p.tatam@cranfield.ac.uk
}

\begin{abstract}
An imaging fibre bundle is incorporated into a full-field imaging OCT system, with the aim of eliminating the mechanical scanning currently required at the probe tip in endoscopic systems. Each fibre within the imaging bundle addresses a Fizeau interferometer formed between the bundle end and the sample, a configuration which ensures down lead insensitivity of the probe fibres, preventing variations in sensitivity due to polarization changes in the many thousand constituent fibres. The technique allows acquisition of information across a planar region with single-shot measurement, in the form of a $2 \mathrm{D}$ image detected using a digital CCD camera. Depth scanning components are now confined within a processing interferometer external to the completely passive endoscope probe.

The technique has been evaluated in our laboratory for test samples, and images acquired using the bundlebased system are presented. Data are displayed either as en-face scans, parallel to the sample surface, or as slices through the depth of the sample, with a spatial resolution of about $30 \mu \mathrm{m}$. The minimum detectable reflectivity at present is estimated to be about $10^{-3}$, which is satisfactory for many inorganic samples. Methods of improving the signal-to-noise ratio for imaging of lower reflectivity samples are discussed.
\end{abstract}

Keywords: OCT, coherence tomography, imaging bundles, coherent fibre bundles

PACS codes: 87.57.-s, 42.30.Wb, 42.81.Qb

\section{Introduction}

Optical coherence tomography (OCT) is a medical imaging technique employing low-coherence interferometry to image the morphology of biological tissue or non-biological semi-transparent composite materials. Illumination wavelengths in the visible and near infra red regions of the spectrum, are strongly scattered by tissue, limiting the maximum imaging depth to about $1 \mathrm{~mm}^{1}$. However, the depth resolution, ranging from about $1 \mu \mathrm{m}$ in ultra-high resolution systems to around $30 \mu \mathrm{m}$, is superior to that achieved by other tomographic methods such as ultrasound, enabling cell-level detail to be observed in the highest resolution systems ${ }^{2}$. OCT is therefore of interest for both in-vitro and in-vivo investigations of epithelial tissues $^{3,4}$. Its ability to perform 'optical biopsy' gives it the potential to replace, or at least to reduce the necessity for, invasive screening by excision of tissue samples ${ }^{5}$.

Laboratory testing can be conveniently performed using bulk-optic OCT instrumentation, but the acquisition of in-vivo images, typically from within the gastro-intestinal tract, requires the use of a flexible, endoscopic probe. This is realized by incorporating optical fibre components. In early systems, the typical configuration comprised a Michelson interferometer formed from a directional coupler ${ }^{6}$. Until recently, time-domain implementations have predominated. In time-domain OCT, broadband illumination is used, and the pathlength of the reference arm is scanned to locate occurrences of the coherence function at a series of sample depths. Optical circulators and balanced detection are now generally used to optimize performance in these systems. The time-domain technique is, however, gradually being superseded by Fourier-domain OCT, which offers superior signal-to-noise performance. In Fourier-domain OCT, the full sample depth is 
addressed simultaneously, the signal beam being dispersed into a sequence of spectral components which carry the structural information.

The majority of bulk and fibre-based OCT systems currently under investigation are based around a Michelson interferometer configuration. This allows an instrument to be constructed using relatively few components and provides good signal-to-noise performance 7 . However, optical fibre versions of the Michelson configuration are susceptible to signal fading caused by bending-induced differences between the states of polarization (SOP) of light in the reference and signal $\mathrm{arms}^{8}$. This is evidently more likely to be a problem during in-vivo measurements. Phase drifting of the fringes, caused by temperature fluctuations, is also likely under these conditions. Previous work in our laboratory has demonstrated that, for fibre-based OCT systems, the Michelson interferometer configuration may not always be the optimum choice. An alternative Fizeau interferometer configuration offers the advantage of down-lead insensitivity ${ }^{9,10}$. Discussion of our current Fizeau OCT system has been previously reported ${ }^{11}$.

The usual procedure in OCT is to scan the position of the focused probe beam rapidly across the sample surface (x-y scanning) to build up a 3D map of tissue structure ${ }^{12}$. In this paper, we discuss the use of a coherent fibre bundle, sometimes known as an imaging bundle, to acquire 3-dimensional volume information without the need for $x-y$ scanning ${ }^{13,14}$. The fibres within a coherent bundle are arranged in a regular array throughout the entire length of up to several metres ${ }^{15}$. An image projected onto one polished end of the bundle is thus transmitted undistorted to the opposite end. In the bundle-based OCT system, reciprocal imaging occurs between the distal end of each fibre in the bundle and the sample, enabling OCT information to be transmitted simultaneously from many positions across the area of the sample illuminated by light from the bundle. Detection of the optical signal over the area addressed by the bundle is performed by a digital camera array, allowing a 3D image to be constructed from a single depth scan.

\section{Time-domain OCT}

Our full-field experimental systems to date have used time-domain processing. Broadband illumination was supplied by super-luminescent diode (SLD) sources at $830 \mathrm{~nm}$. Optimum wavelengths for tissue are those in the range $1000-1550 \mathrm{~nm}$, where absorption of light by the tissue is at its lowest ${ }^{16}$. However, for full-field measurements, silicon technology cameras are much more readily available, with sensitivity in the visible and very near IR, hence the choice of $830 \mathrm{~nm}$ wavelength during development of the new technique.

The structure that appears in an OCT signal results from refractive index discontinuities at boundaries between tissue structures. In a time-domain system, illumination is provided by a broadband non-laser device. The output spectrum should be as broad as possible, since depth resolution is determined by the coherence length, which is inversely related to source spectral width. For a typical super-luminescent source, the spectral width is about 20-100 nm, resulting in a coherence length (and depth resolution) of 10-20 $\mu \mathrm{m}$. A typical single-spot scanned OCT system consists of a Michelson interferometer in which the reference and sample arms are of closely similar length, as shown in figure 1.

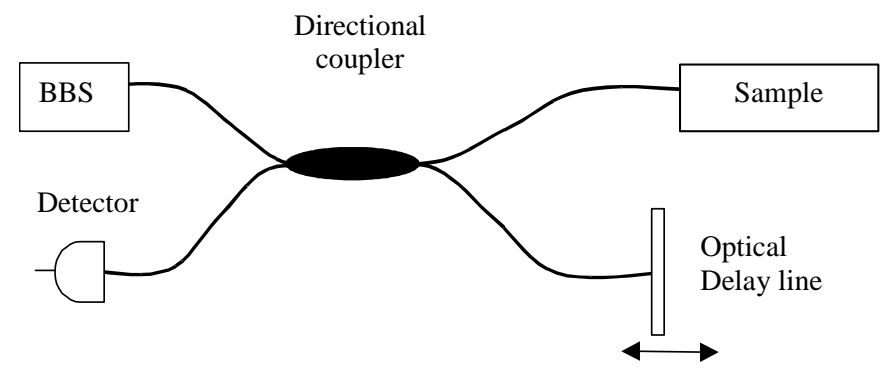

Figure 1. Michelson-configuration OCT system, based on a directional coupler. BBS = broad band source. 
The reference arm includes a variable optical delay line, enabling the reference beam path to be swept linearly over a range of several millimetres. For a tissue boundary at a particular depth within the sample, interference fringes will be seen only when the reference beam path is set to match that of the signal beam, to within the 10-20 $\mu \mathrm{m}$ coherence length of the source. When this condition is satisfied, interference fringes are detected, modulated by the source spectral envelope. Thus the positions of the various tissue boundaries can be located and mapped with high resolution as the reference path is scanned.

Spatial resolution in the plane normal to the optical axis is determined by the spot size of the focused beam within the sample, and is typically similar to the depth resolution. It is determined by the quality of the probe head focusing optics.

The refractive indices of biological materials within a sample of plant or animal tissue are typically rather similar, often differing in only the second or third decimal place and resulting in low reflectivity values. Balanced detection is therefore widely used to improve SNR performance.

\section{Fizeau-configuration OCT using a coherent optical fibre bundle}

For single-spot OCT instruments, 3D information is obtained by rapid scanning of the probe beam, both through the sample depth and in a pattern across the sample surface ${ }^{12}$. This is a reliable technique, relatively straightforward to implement in bulk-optic systems, and typically allows a 3D image of a volume up to a few cubic millimetres to be obtained in a few seconds. However, when using this type of system endoscopically, for internal in-vivo measurements, active scanning is required at the endoscope tip. Although this has been successfully demonstrated using miniaturised components ${ }^{17}$, it requires rather sophisticated engineering, especially for 3-D measurement. A passive probe, that eliminated the need for scanning, would therefore be advantageous for endoscopic use.

A coherent fibre bundle, by allowing simultaneous OCT measurement from an array of positions across the sample, eliminates the requirement for $\mathrm{x}-\mathrm{y}$ scanning ${ }^{13}$. The bundle diameter of a few $\mathrm{mm}$ is suitable for endoscopic use and a fibre spacing as little as $6-10 \mu \mathrm{m}$ can be obtained. The potential spatial resolution is therefore similar to that obtained from an x-y scanned system.

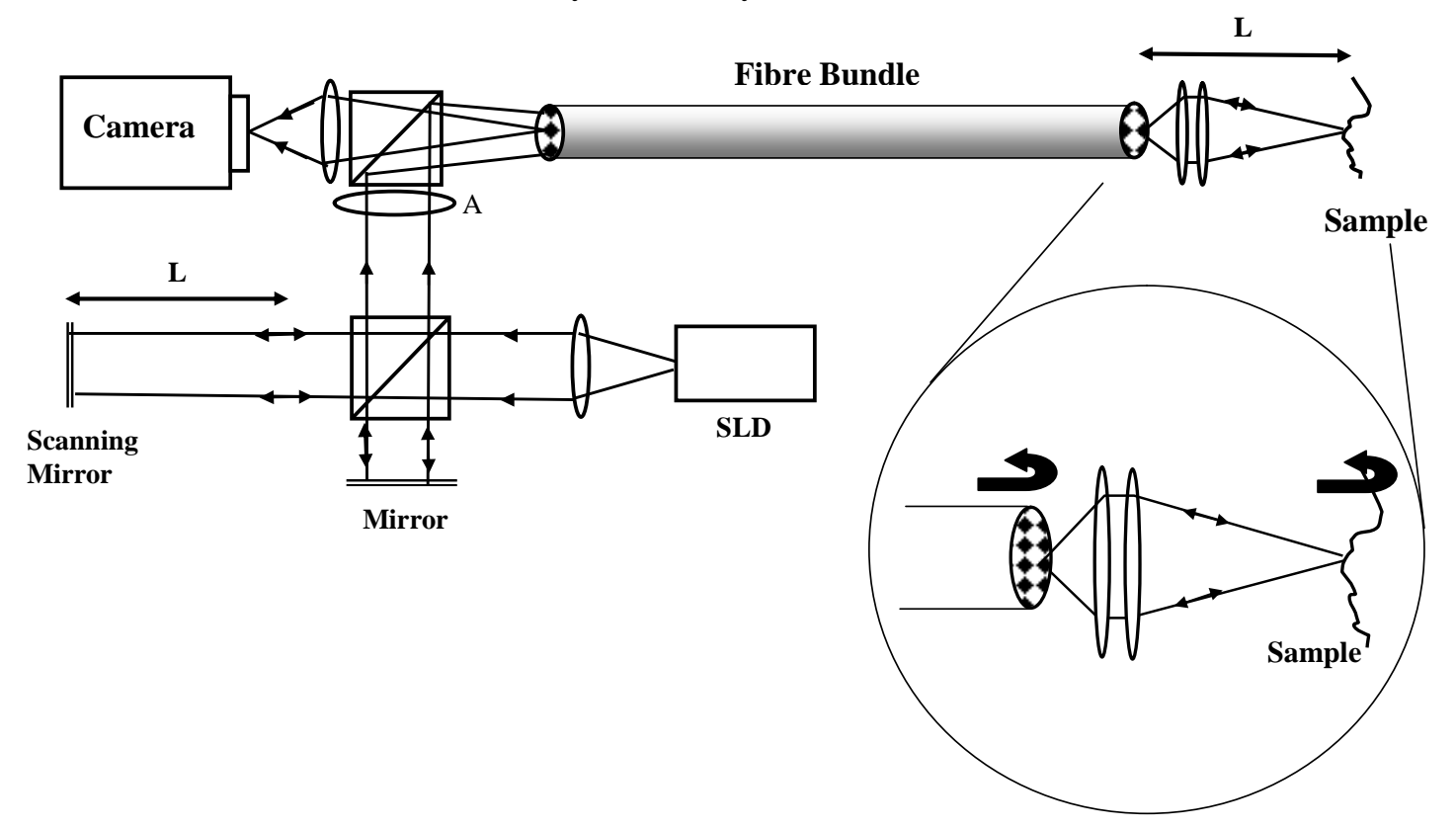

Figure 2. Configuration for 3D OCT employing a fibre imaging bundle. SLD = super-luminescent diode, $A=$ optional focusing lens controlling illumination onto bundle face. The average path-length imbalance $\mathrm{L}$ is identical in both the Fizeau and Michelson interferometers. Inset circle shows where the two reflections occur in the Fizeau interferometer. 
The original configuration, used for our bundle-based OCT system and shown in figure 2, is a full-field version of an arrangement previously employed in our laboratory for a single-point system based on a Fizeau-type probe interferometer. The Fizeau cavity is formed between the end face of the bundle and the sample itself. One related system has been described in the literature, based on a Michelson interferometer. Although this also uses a fibre imaging bundle, it does not employ simultaneous illumination ${ }^{14}$.

Path length matching cannot be achieved directly with the Fizeau arrangement, so a scanned, bulk-optic processing interferometer is employed, having an average path-length mismatch identical to that of the Fizeau. In the work presented here, the processing interferometer is of the Michelson type, but other configurations are possible.

The Fizeau arrangement was considered particularly appropriate for a bundle-based system, in that the bundle itself does not form part of either the sample or the processing interferometer. This gives the instrument 'down-lead insensitivity'. Environmentally-induced polarisation changes within the bundle do not affect the visibility of the interference signal. Because the Michelson processing interferometer uses bulk components, polarisation changes in this section are also avoided. Polarisation-induced fading is a potential problem in any non common-path interferometer, although for single-fibre systems, if not too fast, it can be compensated using polarisation state controllers. In an imaging bundle the Fizeau configuration is a particularly attractive option since it is likely that polarisation is affected differently by each fibre, precluding compensation.

There are, however, disadvantages in using this arrangement, in the form of reduced SNR performance resulting from the high dc signal component when compared with a Michelson interferometer. The implications of this problem are discussed further in section 5 below.

\section{Experimental details}

The experiments described in this paper were performed using the configuration shown in figure 2 . The source was a $7 \mathrm{~mW}, 830 \mathrm{~nm}$ super-luminescent diode (SLD), with a bandwidth of about $15 \mathrm{~nm}$, corresponding to a coherence length of about $30 \mu \mathrm{m}$.

Light from the source was collimated and transmitted through the processing interferometer to the input face of the imaging bundle, via a beam-splitter cube which allowed $50 \%$ of the returning light to be directed onto the detector. A focusing lens (A), positioned after the interferometer, enabled the beam diameter to be adjusted to illuminate any required area of the bundle face. Because the interferometer has unequal path lengths in the two arms, positioning the lens before the interferometer would cause a wavefront curvature mismatch, resulting in bulls-eye fringes in the recombining beams. Backscattered light from the sample reentered the bundle, where it combined with a reference reflection derived from the distal end of the bundle.

The OCT interference signal derives from mixing between sample reflections that have taken the shorter path in the processing interferometer, and a reference reflection that has taken the longer path in the processing interferometer, respectively.

In the configuration shown, light passes first through the processing interferometer, then through the Fizeau system. This allows beams to be collimated through the bulk-optic section, avoiding the formation of circular fringes. Unexpanded beams could be used in this section if required to allow, for example, small aperture modulation components to be included in the interferometer. In this case, a beam expander might be necessary following the Michelson interferometer. In principle, an alternative arrangement could be employed, with the processing interferometer positioned after the Fizeau interferometer. However, the proximal face of the bundle would then have to be imaged through each of the two interferometer arms on the camera. Since the arms are of unequal length, additional optics would be required to compensate for the resulting magnification mismatch. It is likely that difficulties would be encountered in overlaying the two images on the camera with sufficient precision to observe high quality OCT signals.

\subsection{Imaging bundles}

Two types of fibre imaging bundle (supplied by Schott Fiber Optics Inc.) were used in this work. Initially, a wound bundle, $2 \mathrm{~m}$ in length and made up of $300 \times 300$ fibres was employed. The bundle is comprised of 
many hundred "multifibres" wound on a mandrel. The multifibres are groups of 5 x 5 individual fibres assembled on a $10 \mu \mathrm{m}$ centre spacing in a square-packed arrangement, as shown in figure 3(a). The ends of the bundle are fused to produce a rigid section enclosed in a ferrule, while the centre portion remains flexible. Bundles are purchased as a component of selected length with both ends polished to a high-quality optical finish.

Subsequently, leached bundles were also investigated. These are manufactured by acid-leaching away the secondary cladding material from the central region of a drawn multifibre rod, leaving rigid sections at each end for mounting the bundle. These bundles have the advantage of an extremely regular, hexagonal arrangement of the component fibres (figure 3(b)), but are generally available in shorter lengths than the wound bundles. For most OCT applications, this would not be a problem. We found both types of bundle to be appropriate for OCT imaging, having similar loss coefficients, numerical apertures and inter-fibre isolation characteristics.

Light was transmitted by the bundle to a probe head comprising a pair of matched lenses positioned in a unit magnification, $4 f$ imaging arrangement. Spherical lenses were used initially, but aspherics were later introduced to optimise imaging quality, since minimising aberrations across the entire image is of critical importance in the bundle-based system.

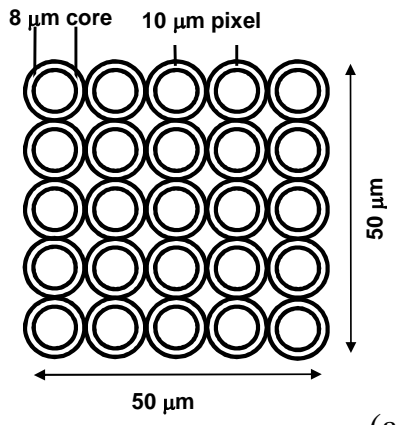

(a)
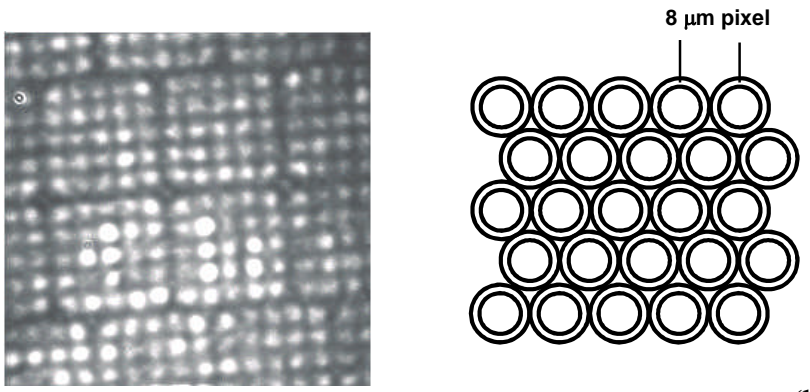

(b)

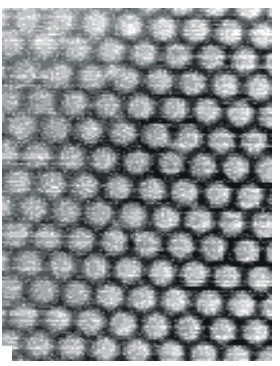

Figure 3. Diagrams and photographs of illuminated bundle end faces, showing the fibre packing arrangements for the Schott imaging bundles used in our laboratory: (a) 5 x 5 square-packed "multifibre" and photograph showing multifibres in a wound bundle. (b) Hexagonal packing used in the leached bundles.

In a single point OCT system, the beam waist diameter determines the spatial resolution of the instrument in the $x-y$ plane normal to the beam direction. Poor focusing degrades spatial resolution. In the bundle-based system, this is similarly true. However, in the full-field system there is an additional consequence of poor focusing. If the beam waist diameter exceeds the fibre spacing, overlap gives rise to inter-fibre cross-talk in light scattered by the sample. Non-reciprocal imaging can occur, in which backscattered light transmitted to the sample by one fibre can be collected by a different fibre. Even if beam waists are well separated at best focus, cross-talk will arise outside the Rayleigh range as the beams diverge.

Since the path length in the Fizeau varies depending on which pair of neighbouring fibres are involved, spurious sets of interference fringes can arise at the detector. Figure 4(a) shows a plot of intensity variation for four individual fibres, randomly located within an illuminated region of about 50x50 fibres, as the processing interferometer is scanned, when probe-head imaging is performed by a pair of simple planoconvex lenses. Multiple fringe bursts can be seen arising from severe cross-talk. The consequences are the appearance of 'ghost' features, causing blurring of interfaces in the OCT image and degradation of both depth ( $\mathrm{z})$ and $\mathrm{x}-\mathrm{y}$ resolution. It can be seen that the precise form of the envelope function in this situation varies from fibre to fibre.

Substitution of achromat lenses resulted in a significant improvement, although their ability to minimise aberrations is known to decrease for off-axis object points. Aspherics performed better still. By choosing an appropriate aperture diameter for our system, focusing quality with aspherics confined about $90 \%$ of the incident power to a single fibre, with about $1-2 \%$ in each of the neighbouring fibres. 

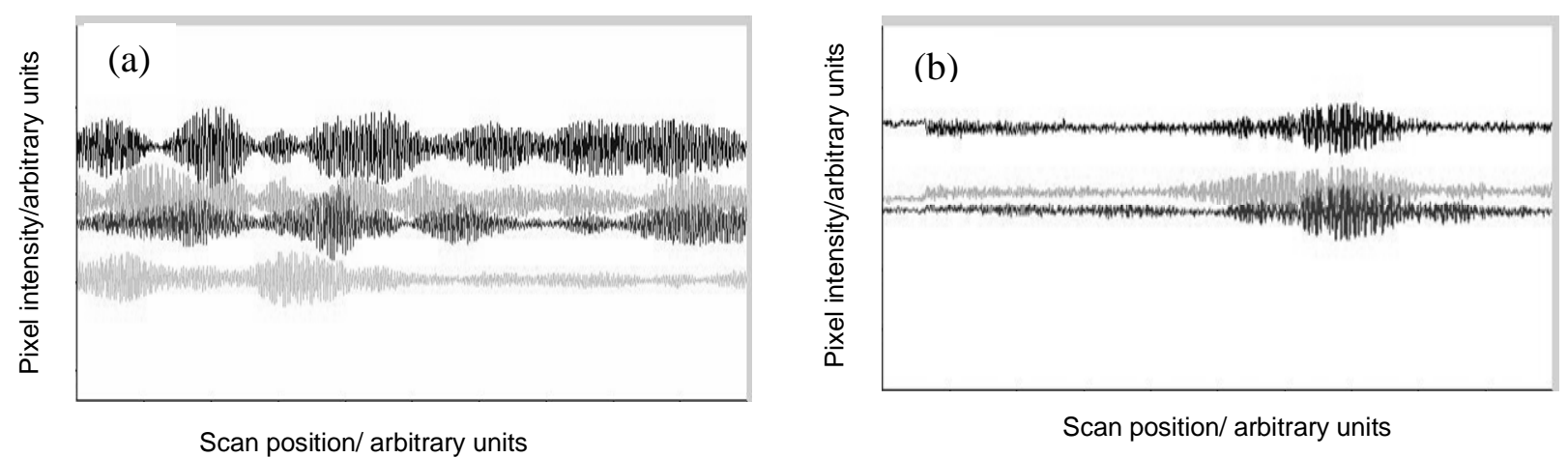

Figure 4. Intensity modulation of the signal from individual fibres in the imaging bundle as the processing interferometer is scanned, when using (a) standard plano-convex and (b) aspherical lenses for focusing in the probe head. The $\mathrm{x}$-axis is on the same scale in each plot.

Typically, no more than one additional pair of interference envelopes was seen to either side of a true interference signal, and the magnitude of the satellite envelopes was reduced to only a few percent of the main envelope. Figure 4(b) shows the intensity modulation for three individual fibres within the illuminated region when aspherics replace the standard lenses in the probe head. The exact position of the envelope maximum varies by a few micrometres for different fibres. This is not unexpected, as the Fizeau cavity length will be dependent upon the flatness of the bundle end face and by the precision of its alignment parallel to the sample surface.

Because the bundle is external to the interferometers, any slight variation in the internal physical length or optical path difference for different regions of the bundle is unimportant. The numerical aperture of the fibre bundle, however, is large, at 0.63 . Under coherent illumination from a laser, the fibres in the imaging bundle are seen to be capable of supporting multiple spatial modes. In principle, 'ghost' images could be seen for light travelling to and from the measurement region in different modes, but under the coupling conditions used in our system, single-mode performance was achieved. The fill-factor for the wound bundle used in this project, in which the basic packing arrangement of fibres was a square grid, was about $30-40 \%$. This is because data is collected only from the fibre core area, with the regions between fibres appearing dark. Use of a bundle therefore gives a somewhat pixelated appearance to the images. The spatial resolution, however, is high, and interpolation or slight smoothing can be used to improve the visual appearance of the OCT images.

\subsection{Detection and processing}

The detector was a 12-bit digital camera (LaVision) with a 640 by 480 pixel array and a variable integration time. The proximal face of the fibre bundle was imaged, via a beam-splitter, onto the image plane of the camera, typically with a magnification of about 4 to 5 . Thus, in the image, a single fibre occupied an area of 16-25 pixels. OCT data were acquired from the camera by downloading successive frames into image buffers as the mirror in the processing interferometer was scanned over a distance of typically $0.2-2 \mathrm{~mm}$.

Data were processed and displayed either as 2D slices, showing $x-z$ planes (depth slices) or $x-y$ (en-face) planes parallel to the sample surface, as shown in figure 5. Slices stored in either orientation can be stacked to produce a full 3D volume image, although the visual interpretation of this is more difficult. The sampling interval was selected to provide data at 300-400 depth positions over a total depth scan range of about $600 \mu \mathrm{m}$, allowing for each fringe envelope encountered to be sampled at least ten times with the source used.

4.2.1 Sequential subtraction. The OCT signal cannot be isolated from the dc component prior to digitization, because digitization takes place within the camera. There is no access to the analogue signal for predigitization filtering. The presence of the dc background places a limitation on the SNR for direct imaging of 
the low-contrast fringes typical in OCT. The OCT signal can, however, be isolated following acquisition, by appropriate combination of images acquired under different phase conditions.

In $x-y$ mode, sample interfaces can be identified by applying a sequential subtraction algorithm to pairs of incoming images, and monitoring the difference value obtained for each pixel. When this value reaches a maximum, corresponding to the peak of a fringe envelope, the scan position is recorded in the final output buffer $^{18}$. A number of samples were investigated, composed of combinations of mirrored and glass surfaces in air.

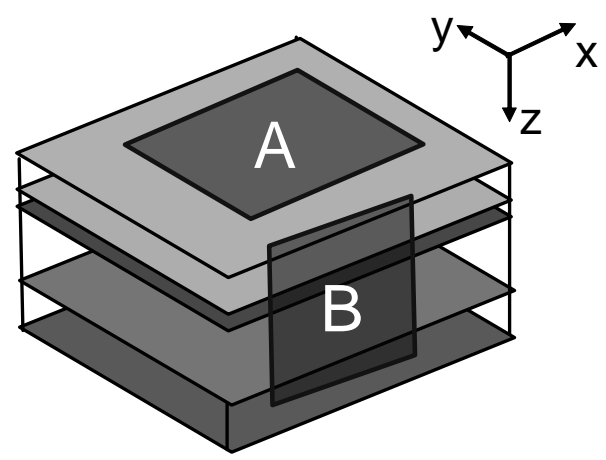

Figure 5. Schematic of sample volume showing the coordinate system used and the image orientation for (A) en-face and (B) depth slice imaging.

\subsubsection{Modulated acquisition with phase-binning}

If the sampling interval is much smaller than the OCT fringe period, sequential subtraction is simple to implement, as acquisition need not be synchronised with z-scanning. However, although this method locates the peak of the OCT envelope, it does not accurately measure the amplitude. We therefore investigated a 4step 'phase-binning' scheme, described by Dubois et $a l^{19}$, implemented by modulating the path length sinusoidally, using a piezo-mounted mirror, and pulsing the source synchronously with a duty cycle of $25 \%$. This allows sets of images to be acquired that correspond to intensity integration over each of the four quarters of the applied modulation period ${ }^{19}$.

The variation in optical intensity I with time, at any camera pixel, is given by

$$
I=I_{0}+A \cos (\phi+\psi \sin (2 \pi f t+\theta))
$$

where $I_{0}$ is the average intensity, $A$ the amplitude of the OCT signal, $\psi$ and $f$ the amplitude and frequency respectively of the path-length modulation, and $\phi$ and $\theta$ are initial phase values.

The pixel intensity values for the four images collected during each quarter of the modulation cycle are denoted $E_{i}$, where $\mathrm{i}=1,2,3,4$. Application of the Bessel function expansions $\Gamma_{\mathrm{s}}$ and $\Gamma_{\mathrm{c}}$ for the integrated intensity values obtained using (1) enables the dc part of the signal to be rejected, by straightforward arithmetic combinations $\Sigma_{s}$ and $\Sigma_{c}$ of the four images, as follows:

$$
\begin{aligned}
& \Sigma_{s}=-E_{1}+E_{2}+E_{3}-E_{4} \propto \Gamma_{s} A \sin \phi \\
& \Sigma_{c}=-E_{1}+E_{2}-E_{3}+E_{4} \propto \Gamma_{c} A \cos \phi
\end{aligned}
$$

For the particular values $\psi=2.45$ and $\theta=0.96$, the two Bessel function expansions $\Gamma_{\mathrm{s}}$ and $\Gamma_{\mathrm{c}}$ are equal, allowing the interferometer phase to be eliminated and the envelope of the OCT signal to be measured directly. The resultant image is, in fact, proportional to the square of the OCT envelope amplitude.

$$
\Sigma_{s}^{2}+\Sigma_{c}^{2}=2 \Gamma_{s}^{2} A^{2}
$$

\section{SNR calculations}

When assessing a conventional OCT system using high-bandwidth photodetectors, a signal-to-noise analysis based upon the contributions from photon noise, detector noise and excess noise is required. For a full-field system using a CCD camera, we monitor accumulated charge rather than a continuous photocurrent, and 
detection is essentially a photon-counting process. The bandwidth filtering and balanced detection techniques that can be applied to improve SNR when using photodetectors are not available in the case of camera detection. A large dc background will dominate the acquired images, with superimposed low-contrast OCT fringes.

The SNR calculation for camera acquisition takes account of the camera dark noise and readout noise, and the Poisson distributed photon noise on the optical signal. When using the four-step binning algorithm described in section 4 , the resulting expression for the signal-to-noise is ${ }^{19}$

$$
S N R=\frac{\gamma^{2}}{2(1+\gamma)} N \xi_{\max }
$$

Where $\mathrm{N}$ is the number of images acquired for each of the four phase ranges, $\xi_{\max }$ is the full-well capacity of the camera $(32,000$ electrons for our camera) and $\gamma$ is the fringe contrast, given by

$$
\gamma=\frac{2 \sqrt{R_{r e f} C R}}{R_{r e f}+C R+2 R_{i n c}}
$$

Here $R_{r e}, R$ and $R_{i n c}$ are the reference reflectivity, signal reflectivity and proportion of incoherent background at the detector respectively. $\mathrm{C}$, in our system, is the recoupling efficiency in the Fizeau cavity. Typically more than $70 \%$ of the light is currently lost in re-coupling in the Fizeau section, because NA matching between the bundle and the focusing optics is rather poor. This could be improved by using custom lenses to design a probe with appropriate input and output NA values. Although more costly, a telecentric probe system, offering a very long Rayleigh range, would be the ideal solution. A factor of $C \approx 0.3$ is included as a multiplier for the sample reflectivity in our current system, to account for recoupling losses.

In a Michelson interferometer system the incoherent signal is generally less than $1 \%$, assuming stray reflections from fibre ends and bulk components have been suppressed. In the Fizeau system, again assuming suppression of the bundle front-face reflection, the main contribution to the incoherent signal is a large dc component resulting from the dual interferometer arrangement. Light which has travelled through both interferometers, but is not path-length matched, makes no contribution to the OCT signal. This fraction is given by

$$
2 R_{\text {inc }}=R_{\text {ref }}+C R
$$

The factor of two arises because $R_{\text {inc }}$ is defined at the detector, at which position half of the light returning from reflections in the Fizeau interferometer has been lost. The minimum detectable reflectivity can be defined by a SNR of 2 . This leads to

$$
R_{\text {min }} \approx \frac{4 R_{r e f}}{N \xi_{\text {max }} C}
$$

For $R_{\text {ref }}=0.04$ and $\mathrm{C}=1$, this suggests a theoretical minimum detectable reflectivity of about $5 \times 10^{-6}$, or -53 $\mathrm{dB}$ when $\mathrm{N}=1$. Taking into account fibre recoupling losses, and the fact that most pixels in are not optimally illuminated, alters this value to a figure closer to $10^{-4}$, or $40 \mathrm{~dB}$.

The camera noise contributes significantly to the total noise. We used a 12-bit camera with a full well capacity of 32,000 electrons. The theoretical dark charge noise was typically 20-100 electrons at integration times used, and the quoted readout noise about 12 electrons. The total measured rms variation on any pixel due to camera noise was about 50 electrons, which will further degrade the SNR from the theoretical value. We would expect, taking this into account, to detect a minimum reflectivity somewhere between $10^{-4}-10^{-3}$ ( -40 to- $30 \mathrm{~dB}$ ) with the current system, corresponding to a fringe contrast of around 0.06 in the original camera images.

SNR can be increased by accumulation of multiple images at each depth position. Accumulating images, although it slows down the acquisition process, should lead to a significant improvement in the theoretical SNR. Averaging 10 images for each scan position would be expected to improve the minimum detectable reflectivity by about $10 \mathrm{~dB}$ (to between -50 and $-40 \mathrm{~dB}$ ), sufficient for some biological applications and many engineering applications. The theoretical SNR also would be increased, for optimally illuminated 
pixels, by using a cooled camera with a larger full-well capacity and lower noise. Illumination with a nearGaussian beam profile will clearly result in optimal performance only close to the centre of the image. A tophat illumination profile would be preferred.

\section{Results}

The ability of the system to detect surfaces of varying reflectivity was assessed by interposing a $200 \mu \mathrm{m}$ thick microscope cover slip across and in contact with a mirror placed in the sample position, so as to cover about half of the camera image. Figure 6 shows (a) an en-face image, (b) a depth slice image, (c) a representation of the sample and (d) a 3D plot generated from the en-face image with slight smoothing applied using a $3 \times 3$ median filter. The results presented in figure 6 were obtained using simple sequential subtraction processing. The translation stage was not in this case synchronised with acquisition.

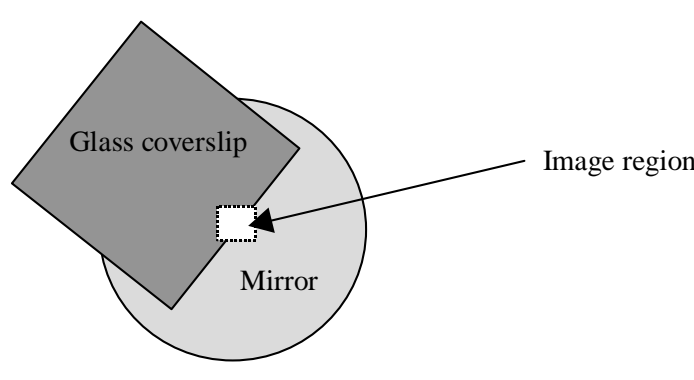

(a)

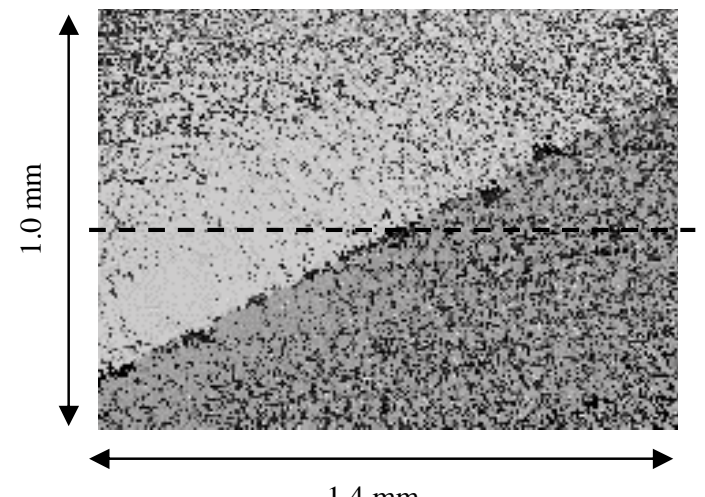

(c)

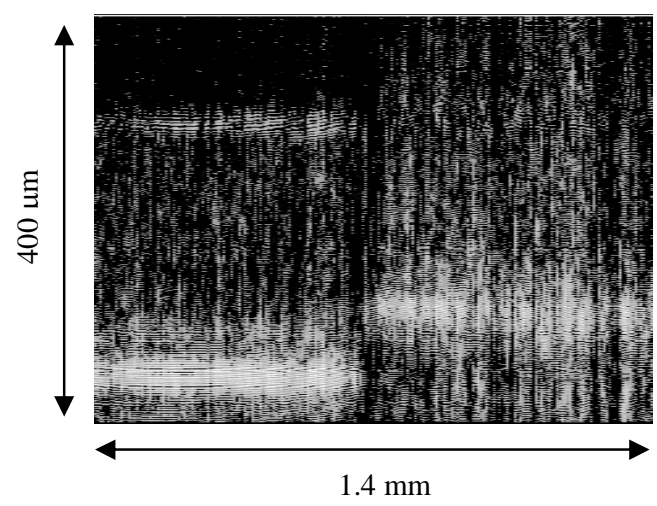

(b)

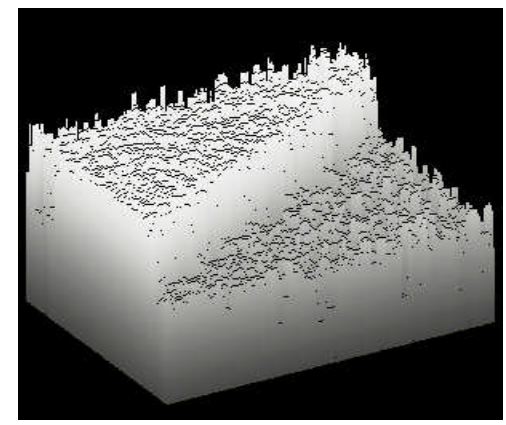

(d)

Figure 6. (a) Representation of the sample composed of a mirror and a glass coverslip. (b) En-face OCT image of sample. (c) Depth slice OCT image corresponding to the position of the dotted line in (b). (d) 3D plot generated from smoothed version of image (a).

The interface corresponding to the mirror surface can be clearly identified in both representations. The portion of this interface that is in contact with the coverslip appears shifted in the images by about $100 \mu \mathrm{m}$. This is because the cover slip increases the optical path length in the Fizeau by a distance $t\left(n_{g}-n_{a}\right)$, where $t$ is the cover slip thickness and $\mathrm{n}_{\mathrm{g}}=1.5$ and $\mathrm{n}_{\mathrm{a}}=1$ the refractive indices of the glass and of air respectively. The sample area addressed is about $1.4 \mathrm{~mm} \times 1 \mathrm{~mm}$, with a scan depth of about $0.4 \mathrm{~mm}$, and the depth slice corresponds to the central row in the en-face image, as shown. 
The glass/air interface at the front of the cover slip produces only a $4 \%$ reflection, and is therefore more difficult to detect than the mirrored surfaces. However, although much fainter than the signals produced from the mirror reflections, it is visible in the depth slice as a second line near to the top of the image.

Following implementation of the 4-step phase-binning processing scheme, further measurements were made to assess the ability of this technique to remove the dc component, and to investigate the effect of accumulating multiple images for each of the four phase ranges. Images of a mirror surface, obtained using accumulations of 1 and 10 frames at each depth, are shown in figure 7.

(a)

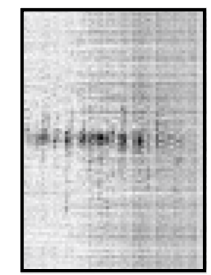

(b)

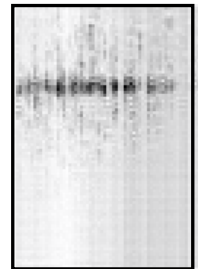

Figure 7. OCT mages obtained from a mirror surface (inverted greyscale) accumulating (a) 1 and (b) 10 frames for each phase range.

Variations in illumination efficiency between fibres complicate calculation of the SNR for the images shown in figure 7. The SNR for the best-illuminated pixels in the first image is about 40 (16 dB), compared with a theoretical SNR of $28 \mathrm{~dB}$ obtained from the analysis in section 5, assuming about half the full-well capacity of the CCD camera to be used for the best-illuminated pixels. The theoretical analysis ignores readout noise, and it is also possible that the recoupling factor used in the theoretical calculation is over-optimistic. The SNR with 10 accumulations increases to about $100(20 \mathrm{~dB})$. The improvement in contrast is clearly visible in the image using a higher number of accumulated frames. However, the improvement is less than would be expected for the number of accumulations used, which suggests that part of the residual signal results from coherent mixing and is not eliminated by the averaging process.

If a display linear with signal value is used, the visual dynamic range is limited to $36 \mathrm{~dB}$, therefore it is preferable to use a non-linear scale that compresses the data range. In this case, the square root of the images has been taken before display. The current SNR is insufficient to obtain an image from tissue, due in part to the high incoherent signal contribution inherent to the Fizeau configuration, which places a limit on system performance, and in part to an apparent residual coherent signal, which may result from short (tens of microns) reflective cavities within the optical system. .

Figure 8 shows a depth slice OCT image of the surface of a $300 \mu \mathrm{m}$ thick plastic film $(\mathrm{n} \approx 1.4 \mathrm{using} 50$ acquisitions per phase range at each depth position sampled. An inverted greyscale is used in this case. The upper and lower surfaces are plainly visible in the central region of the figure, although the SNR is only just sufficient in the region of less well illuminated pixels towards the periphery.

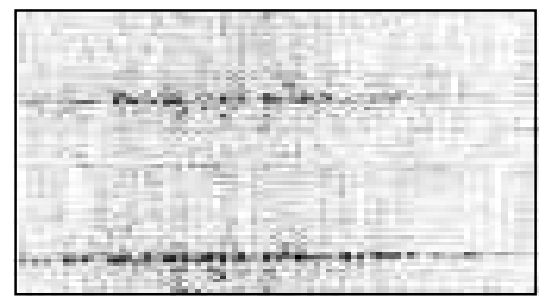

Figure 8. Inverted greyscale image of both surfaces of a $300 \mu \mathrm{m}$ thick plastic film (accumulating 50 frames) 
The value of minimum detectable reflectivity would be improved by increasing the level of illumination to fully utilise the full-well capacity of the camera, and by improving NA matching in the probe section to ensure optimal recoupling to the fibre bundle.

\section{Discussion}

The bundle-based approach to 3D OCT image acquisition has been successfully demonstrated above for laboratory samples of relatively high reflectivity. The elimination of $\mathrm{x}-\mathrm{y}$ scanning allows a completely passive endoscopic probe to be configured. There are two important questions to be addressed. The first is whether the technique has potential for measurements in biological tissue, where reflectivity values are expected to be two or more orders of magnitude lower than those of the current non-biological laboratory samples. The other issue is acquisition speed; can a bundle-based system run at speeds comparable to current systems incorporating fast $\mathrm{x}-\mathrm{y}$ galvo-scanners?

7.1 SNR performance. The signal-to-noise ratio (SNR) of the images shown above is adequate for the measurements made here, but requires significant improvement to allow more complex samples to be studied. One straightforward way to achieve this would be by increasing the power of the source, which currently provides a maximum of only around $7 \mathrm{~mW}$ of optical power. The optical arrangement shown is not optimised for efficiency. Half the light is currently lost in the processing interferometer and a further $50 \%$ each time light passes through the beam splitter at the input of the fibre bundle. The use of a bulk optical circulator, would greatly improve the optical efficiency.

An important factor in optimising depth resolution is to minimise cross-talk between the fibres, which otherwise produces small spurious interferometric intensity oscillations at depth scan positions slightly off the path-balanced condition for a particular sample interface. These arise, as discussed above, when the focused beam-waist at the sample from each fibre becomes larger than the inter-fibre spacing. We are able to reduce inter-fibre cross talk to a negligible level, but as our Rayleigh range is less than $100 \mu \mathrm{m}$, this currently requires very careful positioning of the beam focus with respect to our sample. Improvements to the design of the focusing optics in the probe head, to increase the Rayleigh range, would relax this requirement significantly, and improve SNR for depth positions away from the beam focus.

7.2 Acquisition speed. The processing time currently required in our system to generate an image in either the $\mathrm{x}-\mathrm{y}$ or $\mathrm{x}-\mathrm{z}$ plane is of the order of several minutes. However, this is limited primarily by a slow processing algorithm. The camera is capable of reading out data at 30 frames per second; faster if pixel binning is employed to reduce the amount of data per frame. Thus a set of say 100 images acquired at a sampling interval of $2 \mu \mathrm{m}$ could be obtained within $3 \mathrm{~s}$ or less and stored straight to RAM. Some further time would need to be allocated for post-processing, but 3D OCT information would be obtained in seconds rather than minutes. Current fast systems using galvanometer scanners produce an image in around 1-5 s, so the potential acquisition speed of the camera system approaches that of mechanically scanned instruments.

\section{Conclusions}

An imaging fibre bundle has been employed to construct a full-field OCT system with a passive endoscopic probe section. It has been shown that 3D structural information can be obtained from a sample without any requirement for beam-scanning in the $x-y$ plane normal to the optical axis. The spatial resolution of the bundle-based system compares well with that of active OCT designs using galvanometer mirrors. For the current system, the spatial resolution in the x-y plane and the depth resolution are both about $30 \mu \mathrm{m}$.

Measurements have been successfully made on non-biological surfaces in air, over scan depths of up to about $0.75 \mathrm{~mm}$. The SNR of the system is adequate to detect glass/air interfaces, but is currently insufficient to allow biological samples to be imaged. However, modifications to improve the SNR to the required value have been discussed, including an increase in source power and inclusion of additional optical components to produce a more efficient configuration. Further investigation, following implementation of these improvements, will determine the ultimate quality of the 3D images acquired when bundle-based OCT is used to examine biological tissue. 


\section{Acknowledgments}

Helen Ford acknowledges a Daphne Jackson Research Fellowship sponsored by the Royal Academy of Engineering.

\section{References}

[1] Colston B W, Everett M J, DaSilva L B, Otis L L, Stroeve P and Nathel H 1998 Imaging of hard- and soft-tissue structure in the oral cavity by optical coherence tomography Appl. Opt. 37 3582-85

[2] Drexler W, Morgner U, Kartner F X, Pitris C, Boppart S A, Li X D, Ippen E P and Fujimoto J G 1999 Optical coherence tomography for neurosurgical imaging of human intracortical melanoma Opt. Lett. 24 1221-23

[3] Lazar R, Brunner H and Steiner R 1996 Optical coherence tomography (OCT) of human skin with a slow-scan CCD camera Proc. SPIE 2925 pp 143-51

[4] Bamford K J, James S W, Barr H and Tatam R P 1999 Optical low coherence tomography of bronchial tissue Proc. SPIE 3858 pp 172-9

[5] Bamford K, James J, Barr H and Tatam R P 2000 Radar detection of precancerous bronchial tissue Lasers in Medical Science 15 188-94

[6] Izatt J A, Kulkarni M D, Wang H-W, Kobayashi K and Sivak M V Jr. 1996 Optical coherence tomography and microscopy in gastrointestinal tissues IEEE Journal of Selected topics in quantum electronics 2 1017-28

[7] Rollins A M and Izatt J A 1999 Optimal interferometer designs for optical coherence tomography Opt. Lett. 24 1484-6

[8] Kersey A D, Marrone M J, Dandridge A and Tveten A B 1988 Control scheme for polarization-induced signal fading in multiplexed optical fibre interferometric sensors J. Lightwave Technol. 6 1599-1609

[9] Beddows R, James S W and Tatam R P 2002 Improved performance interferometer designs for optical coherence tomography $15^{\text {th }}$ International Conference on Optical Fibre Sensors (OFS-15) (Portland, Oregon, 6-10 May, 2002) IEEE Cat. No: 02EX533, pp 527-30

[10]Ford H D, Beddows R, Casaubieilh P and Tatam R P 2005 Comparative signal-to-noise analysis of fibreoptic based OCT systems Journal of Modern Optics 52 1965-79

[11]Casaubieilh P, Ford H D and Tatam R P 2005 Optical coherence tomography with a Fizeau interferometer configuration Proc. SPIE 5858-18

[12]Podoleanu A Gh, Dobre G M and Jackson D A 1998 En-face coherence imaging using galvanometer scanner modulation Opt. Lett. 23 147-9

[13] Ford H D and Tatam R P 2005 Full-field optical coherence tomography Proc. SPIE 5858-19

[14]Xie T, Mukai D, Guo S, Brenner M and Chen Z 2005 Fiber-optic-bundle-based optical coherence tomography Opt. Lett. 30 1803-5

[15] http://www.us.schott.com/fiberoptics/english/products/imaging/index.html. (Correct on 20/06/07).

[16]Chance B 1988 Photon Migration in Tissues (New York: Plenum Press)

[17] Tearney G J, Boppart S A, Bouma B E, Brezinski M E, Weissman N J, Southern J F and Fujimoto J G 1996 Scanning single-mode fiber optic catheter-endoscope for optical coherence tomography Opt. Lett. 21 543-5

[18]Balboa I and Tatam R P 1997 Source considerations for low coherence speckle interferometry Optical Inspection and Micromeasurements II (Munich, Germany, 16-19 June, 1997) EOS/SPIE Proc. vol 3098 pp 316-24

[19]Dubois A, Vabre L, Boccara A-C and Beaurepaire E 2002 High-resolution full-field optical coherence tomography with a Linnik microscope Appl. Opt. 41 805-12 